\title{
Diabetic nephropathy and type 1 diabetes mellitus: what is new?
}

\author{
Salwa Hussein Swelam ${ }^{1}$, Hend M Moness ${ }^{2}$ \\ 1- Departments of Pediatrics, Faculty of Medicine, Minia University, Minia, Egypt. \\ 2- Departments of clinical pathology, Faculty of Medicine, Minia University, Minia, Egypt.
}

\begin{abstract}
Introduction

Diabetic nephropathy is the leading cause of renal failure in developed and developing countries. Earlier, more sensitive and specific markers of kidney damage might help diagnose and treat diabetic nephropathy at an earlier stage to prevent the progression to renal failure.
\end{abstract}

\section{Aim of the study}

Is to detect early changes in glomerular basement membrane in children with type1 diabetes mellitus.

\section{Patients and Methods}

This study included 170 children, 120 with diabetes mellitus type 1 and 50 apparently healthy children serving as controls. The study and control group were subjected to complete history taking, through clinical examination. They were also subjected to some laboratory investigations including renal function tests, glycosylated haemoglobin (HbA1c), urinary albumin excretion by detecting albumin creatinine ratio (ACR), glomerular filtration rate and urinary podocalyxin (UPCX) by EIA.

\section{Results}

Our study showed that urinary podocalyxin in diabetic children and adolescents was significantly higher than the control group $(\mathrm{P}<0.000)$, and it correlated positively with glycosylated haemoglobin $(\mathrm{HbA} 1 \mathrm{c})$ and albuminuria $(\mathrm{r}=0.577$ and $\mathrm{P}<0.02)$ and $(\mathrm{r}=0.554$ and $\mathrm{P}<0.000)$. Our results showed that microalbuminuric children had highly significant $\mathrm{U}-$ PCX than normoalbuminuric ones.

\section{Conclusion}

We conclude that urinary podocalyxin could be more sensitive and specific marker of kidney damage than microalbuminuria and thus it could be a useful biomarker for detecting early diabetic nephropathy. Also, U-PCX correlate positively with HbA1c concluding that hyperglycaemic state may cause glomerular damage in early stage of diabetic nephropathy and that duration of diabetes may not play a role in that process.

Keywords Diabetic nephropathy, type 1diabetes mellitus, urinary podocalyxin, albuminuria.

Running Title Glomerular basement membrane and type 1 diabetis mellitus: what is the relation?

\section{Corresponding author}

\section{Salwa Hussein Swelam}

Lecturer of pediatrics, Departments of Pediatrics, Faculty of Medicine, Minia University, Minia, Egypt.

Email: Salwasara@yahoo.com

Mobile: 00201006488972

\author{
geget: The Journal of the Egyptian Society of Pediatric Nephrology and Transplantation (ESPNT) \\ geget https://geget.journals.ekb.eg/ \\ Published by ESPNT http://espnt.net/ \\ Cohosted by Egyptian Knowledge Bank https://www.ekb.eg
}




\section{Introduction}

Diabetic nephropathy occurs in approximately one-third of all people with diabetes and is the leading cause of renal failure in developed and developing countries [1]. Clinically, the first sign of diabetic nephropathy is considered to be microalbuminuria [2]. Although microalbuminuria in diabetic patients is considered to be the best predictor of progression to end-stage renal disease [3]; earlier, more sensitive and specific markers of kidney damage might help diagnose and treat diabetic nephropathy at an earlier stage to prevent the progression to renal failure [1].

The glomerular capillary wall consists of three distinct but closely interacting layers: the fenestrated endothelium, with its glycocalyx; the podocytes, with their interdigitated foot processes and slit diaphragms; and the intervening glomerular basement membrane (GBM) [4]. The presence of microalbuminuria indicates the involvement of all of these three components in filtration-barrier injury [5].

Type 1 diabetics have reduced systemic glycocalyx volume, which coincides with the onset of microalbuminuria [6]. Human podocytes (Pods) have been demonstrated to be functionally and structurally injured in the natural history of diabetic nephropathy ${ }^{[7]}$ Podocalyxin is one of the specific markers for Pods; therefore, the presence of urinary podocalyxin (U-PCX) reflects specific Pod injury that is unrelated to the other two elements (endothelial layer and GBM). It appears reasonable that Pod injury alone is present before the appearance of microalbuminuria [8].

The aim of this work is to detect early changes in glomerular basement membrane in children with type 1 diabetes mellitus.

\section{Patients and methods}

This study included 170 children, divided into 2 groups: Group (1); 120 with diabetes mellitus type -1 and were furtherly subdivided into normoalbuminuric and microalbuminuric groups. Group (2); 50 apparently healthy children serving as controls matched in age and sex. Diabetic children were selected from the Diabetes Clinic at our University Hospital, during the period from May 2014 to January 2017.children aged from 5-18 years and duration of diabetes more than 4 years included in the study. Diabetic children less than 5 years and more than 18 years with duration of diabetes less than 4 years were excluded. Control subjects were chosen to be clinically free. The study and control group included in our study were subjected to complete history taking, through clinical examination with stress on anthropometic measures and blood pressure evaluation.
They were also subjected to some laboratory investigations including renal function tests, glycosylated haemoglobin (HbA1c), urinary albumin excretion by detecting albumin creatinine ratio (ACR) measured in a random urine specimen, Glomerular filteration rate (GFR) was calculated using the original Schwartz equation with a creatinine value: GFR $\left(\mathrm{ml} / \mathrm{min} / 1.73 \mathrm{~m}^{2}\right)=(0.41 \times$ Height in $\mathrm{cm}) /$ Creatinine in $\mathrm{mg} / \mathrm{dl}$ [9] and urinary podocalyxin (U-PCX) by EIA.

Detection of microalbuminuria: a spot urine specimen, using first voided urine in the morning, was collected from each patient to detect ACR (albumin / creatinine ratio). Microalbuminuria is defined as an ACR between $30-299 \mathrm{mg} / \mathrm{g}$ [10]. Assessment of $\mathrm{HbA1c} \%$ as a parameter of glycemic control: HbA1c using resin column chromatography. Kit contents were supplied by TECO DIAGNOSTICS, California; USA. Quantitaive determination of urinary Podocalyxin by EIA [11] [Wuhan Eiaab Science Co., LTD, China]. The data were coded and verified prior to data entry. All statistical analyses were carried out using the Statistical Package for Social Sciences (SPSS/Windows Version 19. 0. 0, SPSS Inc., an IBM Company). Microsoft excel 2003 was used for drawing figures.

\section{Results}

Clinical data of diabetic and control groups illustrated in table 1 showed that there was significant difference in systolic and diastolic blood pressure with $\mathrm{P}$ value $<0.019$ and $\mathrm{P}<0.016$ respectively between the diabetic and control group. As regard GFR, HbA1c and U-PCX: there were statistically high significant difference between both groups $(P<0.000)$. Finally, there was moderate significant difference in serum creatinine $(\mathrm{P}$ $<0.006)$ and ACR $(\mathrm{P}<0.001)$ between both groups.

Correlation between U-PCX (urinary podocalyxin) and duration of DM, GFR, HbA1C and ACR illustrated in table 2 showed that there was significant correlation between U-PCX and $\mathrm{HbA} 1 \mathrm{c}(\mathrm{r}=0.577$ and $\mathrm{P}<0.02)$ and there was high positive significant correlation between U-PCX and ACR ( $\mathrm{r}=0.554$ and $\mathrm{P}<0.000)$ in diabetic children. On the other side, there were no significant correlation between U-PCX and each of: duration of diabetes $(r=0.077$ and $P=0.559)$ and GFR $(r=0.259$ and $P$ $=0.327$ ).

Our results showed that microalbuminuric children (42 children) had highly significant U-PCX than normoalbuminuric (78 children) ones. It also showed that both groups had highly significant U-PCX than the control group (figure 1). Sensitivity and specificity of U-PCX in all patients, normoalbuminuric and microalbuminuric diabetics with sensitivity $76.7 \%$ in all patients, $64.1 \%$ in normoalbuminuric group and $100 \%$ in microalbuminuric group with specificity $100 \%$ in all groups as showed in table 3 . 
Table 1 Clinical data of diabetic and control groups (mean \pm SD)

\begin{tabular}{|c|c|c|c|}
\hline Item & Study group "n=120" & Control group "n=50" & P=value \\
\hline SBP (systolic bl. pr.) & $117.75 \pm 8.65$ & $112.92 \pm 7.64$ & $\mathrm{P}<0.019^{*}$ \\
\hline DBP (diastolic bl. pr.) & $79.08 \pm 6.79$ & $75.41 \pm 4.14$ & $\mathrm{P}<0.016^{*}$ \\
\hline S. creatinine (mg/d) & $0.768 \pm 0.129$ & $0.687 \pm 0.089$ & $\mathrm{P}<0.006^{* *}$ \\
\hline S. urea (mg/dl) & $25.98 \pm 5.68$ & $19.79 \pm 4.90$ & $\mathrm{P}<0.000^{* * *}$ \\
\hline GFR (ml/min/1. 73 $\left.\mathbf{~ m}^{\mathbf{2}}\right)$ & $128.34 \pm 22.27$ & $148.24 \pm 15.36$ & $\mathrm{P}<0.000^{* * *}$ \\
\hline ACR (mg/g creatinine) & $54.28 \pm 37.25$ & $6.12 \pm 3.23$ & $\mathrm{P}<0.001^{* *}$ \\
\hline Hb1AC (\%) & $7.07 \pm 1.55$ & $4.27 \pm 0.57$ & $\mathrm{P}<0.000^{* * *}$ \\
\hline U-PCX (ng/ml) & $1.66 \pm 0.533$ & $0.85 \pm 0.22$ & $\mathrm{P}<0.000^{* * *}$ \\
\hline
\end{tabular}

GFR = glomerular filteration rate $\mathrm{ACR}=$ Albumin creatinine ratio

HbA1c = glycated haemoglobin U-PCX = urinary podocalyxin

* Significant difference ** moderate significance *** high significant

Table 2 Correlation between U-PCX (urinary podocalyxin) and some collected clinical data in diabetic children.

\begin{tabular}{|c|c|}
\hline Item & U. PCX \\
\hline Duration of D. M & $\begin{array}{r}\mathrm{r}=0.077 \\
\mathrm{P}=0.559 \mathrm{n} . \mathrm{s}\end{array}$ \\
\hline eGFR & $\mathrm{r}=0.259$ \\
$\mathrm{P}=0.327 \mathrm{n} . \mathrm{s}$
\end{tabular}

n. $s=$ not significant $*$ significant difference $* * *$ high significance

Table 3 Sensitivity and specificity of U-PCX in all patients, normomicroalbuminuric and microalbuminuric groups

\begin{tabular}{|c|c|c|}
\hline Groups & Sensitivity & Specificity \\
\hline All patients & $76.7 \%$ & $100 \%$ \\
\hline Normoalbuminuric & $64.1 \%$ & $100 \%$ \\
\hline Microalbuminuric & $100 \%$ & $100 \%$ \\
\hline
\end{tabular}




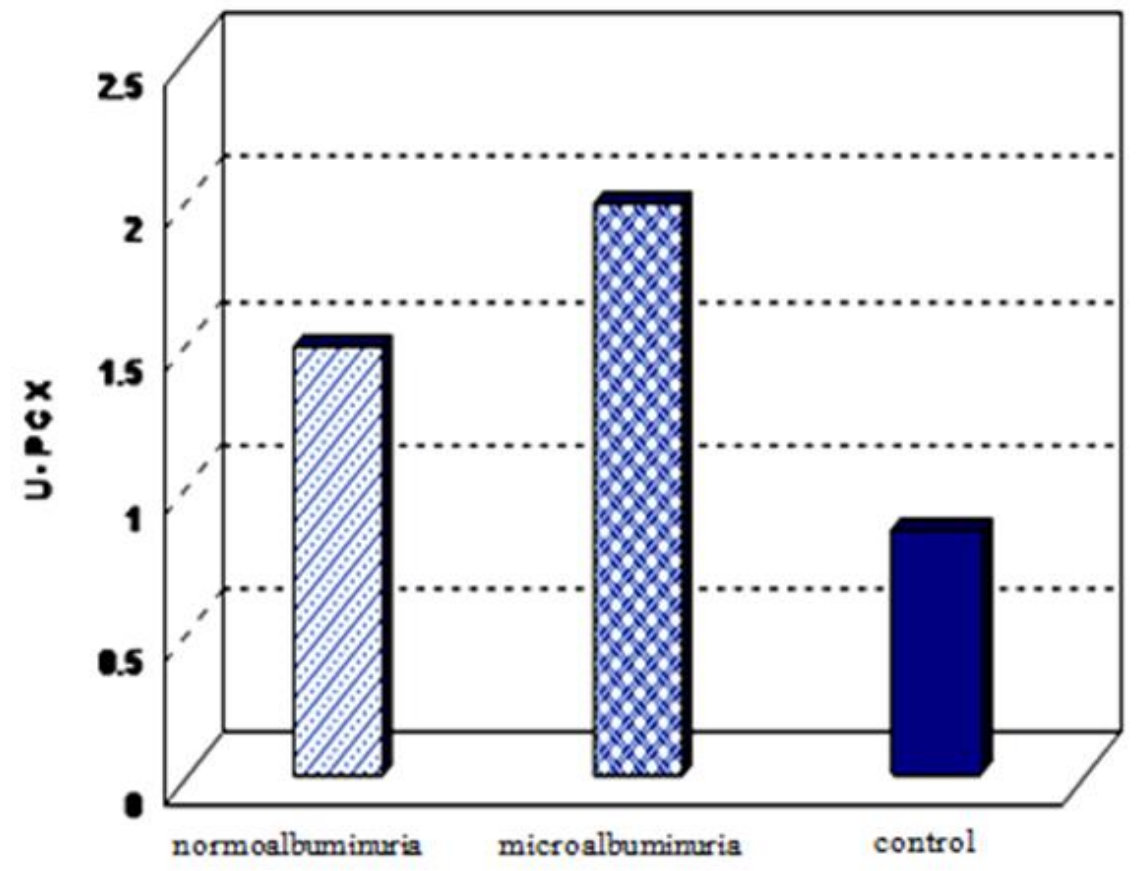

Figure 1 U-PCX in different study groups and control.

\section{Discussion}

Podocytes are key structural elements of the glomerular filtration barrier. It is accepted that podocytes' injuries play an essential role in the progression of diabetic kidney disease [12] in urine can be found in diabetic patients with micro- and macroalbuminuria [13].

In our study, diabetic patients have been divided into normoalbuminuric group and microalbuminuric group according to their urinary albumin excretion measured by ACR to make it easy in studying some correlations.

In our study, we found that children with diabetes had significantly greater U-PCX values than healthy controls which is consistant to Zheng et al, [14] results which revealed that urinary synaptopodin, podocalyxin, CD2-AP, $\alpha$-actin4, and podocin mRNA were significantly increased in DN patients compared with healthy controls

We also found that there were significant positive correlation between urinary podocalyxin and both of: urinary albumin excretion $(\mathrm{ACR})(\mathrm{r}=0.554, \mathrm{P}<0.000)$ and $\mathrm{HbA1c}(\mathrm{r}=0.577, \mathrm{P}$ $<0.02$ ) which is consistant to earlier studies by Jonathan, [15] and Hara et al, [8].

In a study done for patients with type 1 and 2 diabetes, Jonathan [15] found similar significant correlations between podocalyxin and albuminuria $(\mathrm{r}=0.452, \mathrm{P}<0.001)$ and between urinary mRNA expression of podocalyxin and HbA1c $(r=0$. $441, \mathrm{P}<0$. 001), a finding that likely reflects the injury of uncontrolled hyperglycemia and associated mechanisms on the filtration barrier.

Hara et al, [8] in a study done to patients with various glomerular diseases and patients with type 2 diabetes observed the same correlations between U-PCX and HbA1c and urinary albumin levels which might indicate that a hyperglycaemic state causes glomerular capillary -barrier damage as demonstrated by the increased excretion of u-PCX and albuminuria.

In our study we observed that there were no significant correlation between U-PCX and eGFR $(P=0.327)$. In contrast to our study, Zheng et al, [14] observed that estimated GFR correlate negatively with podocalyxin expression $(r=-0.349$, $\mathrm{p}=0.01)$ in a study investigated expression of podocyteassociated genes (as podocalyxin and other genes) in urinary sediment and their relation to disease severity in patients with DN. In his study he used a specific technique (real-time PCR) which has the benefits of excellent sensitivity, quantification, and reproducibility, and it is able to measure low-abundance genes from even one single cell.

In agreement with our results Hara et al, [8] observed the same results as ours and concluded that changes in eGFR typically reflect deranged renal function only in the advanced stages of the kidney disease (when functioning nephrons are lost) not in the earlier stages and if the presence of u-PCX reflects Podocyte injury in the earlier stages of kidney disease, the lack of correlation between eGFR and u-PCX levels is understandable.

Hara et al, [8] found that urinary podocalyxin was higher in 53. $8 \%$ patients at the normoalbuminuric stage, $64.7 \%$ at the microalbuminuric stage and $66.7 \%$ at the macroalbuminuric stage which is consistant to our study which revealed that $64.1 \%$ of patients had high levels of U-PCX at the normoalbuminuric stage above the cut off value.

\section{Conclusion}

We conclude that urinary podocalyxin could be more sensitive and specific marker of kidney damage than microalbuminuria and thus it could be a useful biomarker for detecting early diabetic nephropathy. Also, U-PCX correlate positively with $\mathrm{HbA1c}$ concluding that hyperglycaemic state 
may cause glomerular damage in early stage of diabetic nephropathy and that duration of diabetes may not play a role in that process.

\section{Recommendations}

From our study we recommend that

- Determination of urinary podocalyxin in diabetic children is considered to be a noninvasive technique for early detection of diabetic nephropathy.

- More studies should be done to closely evaluate UPCX in diabetics since beginning of the disease and follow up in different stages of nephropathy.

\section{References}

1. Abraham Cohen-Bucay and Gautham Viswanathan Urinary Marker of Glomerular Injury in Diabetic Nephropathy. International Journal of Nephrology. 2012; 2012: 146987

2. Remuzzi G., A. Schieppati, and P. Ruggenenti. Nephropathy in patients with type 2 diabetes. New England Journal of Medicine. 2002; 346 (15): 114551.

3. Zeeuw D, D. Ramjit, Z. Zhang et al. Renal risk and renoprotection among ethnic groups with type 2 diabetic nephropathy: a post hoc analysis of RENAAL. Kidney International. 2006; 69 (9): 1675-82.

4. George Jarad and Jeffrey H. Miner. Update on the glomerular filtration barrier. Curr Opin Nephrol Hypertens. 2009; 18 (3): 226-33.

5. Patari A, Forsblom C, Havana M, Taipale H et al. Nephrinuria in diabetic nephropathy of type 1diabetes. Diabetes. 2003; 52 (12): 2969-74.

6. Nieuwdorp M, Mooij HL, Kroon J, Atasever B, Spaan JA Hollemet al. Endothelial glycocalyx damage coincides with microalbuminuria in type 1 diabetes. Diabetes. 2006; 55 (4): 1127-32

7. Wolf G, Chen S and Ziyadeh FN. From the periphery of the glomerular capillary wall toward the center of disease: podocyte injury comes of age in diabetic nephropathy. Diabetes. 2005; 54 (6): 1626-34.

8. Hara M, Yamagata K, Tomino Y, Saito A, Hirayama Y et al. Urinary podocalyxin is an early marker for podocyte injury in patients with diabetes: establishment of a highly sensitive ELISA to detect urinary podocalyxin. Diabetologia. 2012; 55 (11): 2913-9.

9. Schwartz GJ and Work DF. Measurement and estimation of GFR in children and adol escents. Clin J Am Soc Nephrol. 2009; 4 (11): 1832-43.

10. KDOQI. Clinical Practice Guidelines and Clinical Practice Recommendations for Diabetes and Chronic Kidney Disease. Am J Kidney Dis. 2007; 49 (2): S12154.

11. Kershaw DB, Beck SG, Wharram BL, et al. Molecular cloning and characterization of human podocalyxinlike protein. Orthologous relationship to rabbit PCLP1 and rat podocalyxin. J Biol Chem. 1997; doi: 10.1074/jbc.272.25.15708.

12. Weil EJ, Lemley KV, Yee B, et al. Podocyte detachment in type 2 diabetic nephropathy. Am J Nephrol. 2011; 33 (S1): 21-4.

\section{List of Abbreviations}

ACR; albumin creatinine

DM; diabetes mellitus

DN; diabetic nephropathy

EIA;Enzyme Immuno Assay HbA1c; glycosylated haemoglobin
GBM; glomerular basement membrane

GFR; Glomerular filtration rate

PCR; Polymerase Chain

Reaction.

Pods; podocytes

U-PCX; urinary podocalyxin

13. Nakamura T, Ushiyama C, Suzuki S, et al. Urinary excretion of podocytes in patients with diabetic nephropathy. Nephrol Dial Transplant. 2000; 15 (9): 1379-83.

14. Zheng M, Lv LL, Ni J, Ni HF, Li Q, Ma KL and Liu BC. Urinary podocyte-associated mRNA profile in various stages of diabetic nephropathy. PLoS One. 2011; 6 (5): e20431.

15. Jonathan Fraportti do Nascimento, Luis H Canani, Fernando Gerchman, Patricia G Rodrigue et al. Messenger RNA levels of podocyte-associated proteins in subjects with different degrees of glucose tolerance with or without nephropathy. BMC Nephrology. 2013; 14 (1): 1-18.

\section{Statements}

\section{Ethics approval and consent to participate}

This study protocol and the consents were approved and deemed sufficient by the Ethical Committee of Helsinki and agreed by the faculty of medicine, Minia university, Ethical committee (No: 116-11-2014) and informed written consent was obtained in every case from their legal guardians.

\section{Consent for publication}

"Not applicable"

\section{Availability of data and material}

"Not applicable"

\section{Conflict of interest}

The authors declare no conflict of interest.

\section{Funding}

The authors declare that this research work did not revise any fund

\section{Acknowledgements}

We would like to thank all patients and their family members for their valuable contributions to the study. 